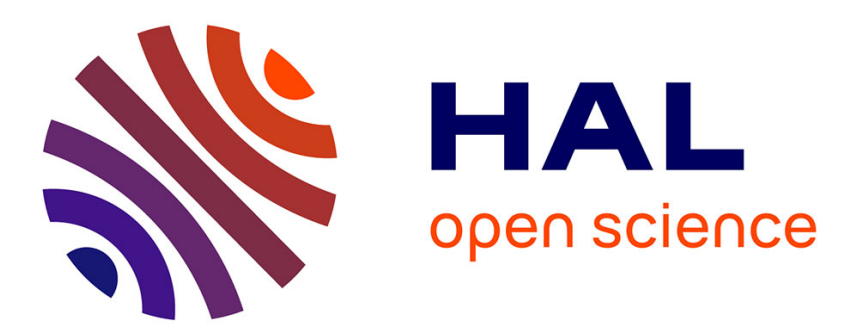

\title{
Robust Probabilistic-Constrained Optimization for IRS-Aided MISO Communication Systems
}

\author{
Tuan Anh Le, Trinh van Chien, Marco Di Renzo
}

\section{To cite this version:}

Tuan Anh Le, Trinh van Chien, Marco Di Renzo. Robust Probabilistic-Constrained Optimization for IRS-Aided MISO Communication Systems. IEEE Wireless Communications Letters, In press, 10.1109/LWC.2020.3016592 . hal-03020451

\section{HAL Id: hal-03020451 https://hal.science/hal-03020451}

Submitted on 23 Nov 2020

HAL is a multi-disciplinary open access archive for the deposit and dissemination of scientific research documents, whether they are published or not. The documents may come from teaching and research institutions in France or abroad, or from public or private research centers.
L'archive ouverte pluridisciplinaire HAL, est destinée au dépôt et à la diffusion de documents scientifiques de niveau recherche, publiés ou non, émanant des établissements d'enseignement et de recherche français ou étrangers, des laboratoires publics ou privés. 


\title{
Robust Probabilistic-Constrained Optimization for IRS-Aided MISO Communication Systems
}

\author{
Tuan Anh Le, Senior Member IEEE, Trinh Van Chien, and Marco Di Renzo, Fellow IEEE
}

\begin{abstract}
Taking into account imperfect channel state information, this letter formulates and solves a joint active/passive beamforming optimization problem in multiple-input singleoutput systems with the support of an intelligent reflecting surface. In particular, we introduce an optimization problem to minimize the total transmit power subject to maintaining the users' signal-to-interference-plus-noise-ratio coverage probability above a predefined target. Due to the presence of probabilistic constraints, the proposed optimization problem is non-convex. To circumvent this issue, we first recast the proposed problem in a convex form by adopting the Bernstein-type inequality, and we then introduce a converging alternating optimization approach to iteratively find the active/passive beamforming vectors. In particular, the transformed robust optimization problem can be effectively solved by using standard interior-point methods. $\mathrm{Nu}$ merical results demonstrate the effectiveness of jointly optimizing the active/passive beamforming vectors.
\end{abstract}

Index Terms-6G wireless, intelligent reflecting surface.

\section{INTRODUCTION}

Intelligent reflecting surface (IRS), i.e., a programmable planar array of passive reflecting elements, has been identified as an energy-efficient technology for improving the performance of wireless communication systems [1]-[4]. An IRS-aided communication system comprises an IRS, a base station (BS) and the mobile users. The location of the IRS is usually chosen to assist the BS communicating with the users. An IRS can also extend the communication range where there is no direct link between the BS and the users. Each element of the IRS can be independently controlled to produce a desired phase shift on the impinging electromagnetic waves. A careful design of the phase shifts of all the elements of the IRS helps to improve the channel propagation conditions [5]. Moreover, the combination of transmit beamforming and phase shift designs has been proved to offer higher spectral and energy efficiencies compared to conventional beamforming methods [6].

Manuscript received March 24, 2020; revised July 09, 2020 and August 07, 2020; accepted August 07, 2020. Date of publication XXX; date of current version XXX. The work of M. Di Renzo was supported in part by the European Commission through the H2020 ARIADNE Project under Grant 871464. The associate editor coordinating the review of this letter and approving it for publication was Dr. J. Xu. (Corresponding author: Trinh Van Chien.)

T. A. Le is with the Department of Design Engineering \& Mathematics, Faculty of Science and Technology, Middlesex University, The Burroughs, Hendon, London, NW4 4BT, U. K. Email: t.le@mdx.ac.uk.

T. V. Chien was with the School of Electronics and Telecommunications, Hanoi University of Science and Technology, 100000 Hanoi, Vietnam. He is now with the Interdisciplinary Centre for Security, Reliability and Trust (SnT), University of Luxembourg, L-1855 Luxembourg, Luxembourg. Email: vanchien.trinh@uni.lu.

M. Di Renzo is with Université Paris-Saclay, CNRS, CentraleSupélec, Laboratoire des Signaux et Systèmes, 91192 Gif-sur-Yvette, France. Email: marco.direnzo@centralesupelec.fr.
The performance of an IRS-aided communication system heavily depends on the accuracy of the channel state information (CSI), i.e., the CSI between the BS and the IRS, as well as the CSI between the IRS and the mobile users. Most of the works on IRS-aided systems are based on a perfect CSI assumption, see e.g., [7] and references therein. Unfortunately, due to the random nature of wireless systems, obtaining accurate CSI is, in practice, a challenging task. Therefore, the robust optimization of the active/passive beamforming against imprecise estimates of the CSI is highly desirable in order to fully realize the potential of IRS-aided communication systems.

The first attempt to tackle imperfect estimates of the CSI in IRS-aided systems can be found in [8]. In [8], the transmit beamforming vectors at the BS and the phase shifts at the IRS are jointly designed so as to minimize the total transmit power subject to the worst-case quality of service $(\mathrm{QoS})$ constraints, i.e., maintaining the required achievable rate for every user under all possible cases of CSI errors. To that end, an ellipsoid approach was adopted to capture the channel uncertainties, where the Frobenious norm of the channel error vector is assumed to be confined within a given radius of the uncertainty region. This method results in a conservative approach since it allocates excessive system resources to satisfy rarely occurring worst-case events.

In this letter, we tackle the problem of allocating excessive system resources by allowing that the QoS constraints can be violated with a certain probability. In particular, we formulate an optimization problem to jointly design the active/passive beamforming vectors at the BS and the IRS. The proposed problem minimizes the total transmit power while maintaining the users' signal-to-interference-plus-noise-ratio (SINR) coverage probability above a predefined value. Since the considered probabilistic constraints are not convex, we adopt the Bernstein-type inequality [9] to transform the formulated problem into a linear matrix inequality (LMI) form, i.e., into a convex optimization problem. In addition, we introduce an alternating optimization approach to iteratively find the active/passive beamforming vectors. Our proposed problem differs from that in [8] for two main reasons. First, the QoS constraints are formulated in a probabilistic form while those of [8] are not. Second, unlike [8], the considered problem does not rely on the assumption of bounded channel uncertainty region.

The following notation is adopted in this letter. Bold lower/upper case letters denote vectors/matrices; $\|\cdot\|$ and $\|\cdot\|_{F}$ denote the Euclidean norm and the Frobenius norm, respectively; $(\cdot)^{H},(\cdot)^{T}$ and $(\cdot)^{*}$ denote the complex conjugate transpose operator, the transpose operator and the complex 
conjugate operator, respectively; $\operatorname{tr}(\cdot)$ denotes the trace of a matrix; $\mathbf{X} \geq \mathbf{0}$ denotes the positive semidefinite condition; $\mathbf{I}_{a}$ denotes an $a \times a$ identity matrix; $\operatorname{diag}(\mathbf{x})$ denotes a diagonal matrix whose diagonal entries are the elements of the vector $\mathbf{x}$; diag $(\mathbf{X})$ denotes a vector comprising the diagonal elements of matrix $\mathbf{X} ; \mathbf{1}_{N}$ denotes an $N \times 1$ vector of all unity elements; $C \mathcal{N}(\cdot, \cdot)$ denotes a circularly symmetric complex Gaussian distribution; $\operatorname{vec}(\cdot)$ denotes the vectorization operator; $\operatorname{Pr}(\cdot)$ denotes the probability of an event; $\otimes$ denotes the Kronecker product; $|\cdot|$ and $\arg (\cdot)$ denote the absolute value and the phase of a complex-valued scalar, respectively; $\mathbb{E}[\cdot]$ denotes the expectation of a random variable.

\section{SySTEM MODEL}

We consider an IRS-aided communication system that consists of an $M$-antenna BS serving $K$ single-antenna users. It is assumed that there is no direct communication link from the BS to the users due to the presence of blockages, e.g., high buildings. To overcome this issue, an IRS with $N$ reflective elements is deployed to assist the communication between the BS and the users. Let $\mathbf{H}=\left[\mathbf{h}_{1}, \ldots, \mathbf{h}_{N}\right] \in \mathbb{C}^{M \times N}$ and $\mathbf{g}_{k}=\left[g_{k 1}, \ldots, g_{k N}\right]^{T} \in \mathbb{C}^{N \times 1}$ denote the channel coefficients between the BS and the IRS, as well as between the IRS and the $k$-th user, respectively. We assume that the instantaneous CSI is not known, and that the channel is estimated from the uplink training data. Therefore, the system operates under a time division duplexing protocol and by leveraging the channel reciprocity of the downlink and uplink channels. ${ }^{1}$

\section{A. Uplink training phase}

In the uplink training phase, $N$ time slots are dedicated to the channel estimation by utilizing $K$ orthogonal pilot signals $\left\{\boldsymbol{\psi}_{1}, \ldots, \boldsymbol{\psi}_{K}\right\}$, each comprising $\tau_{p}$ symbols with $\tau_{p} \geq K$. In each time slot, one reflective element is chosen to only reflect the training pilot signal without introducing any phase shift while the other $N-1$ elements do not reflect the incident signal. $^{2}$ The pilot signal reflected by the $n$-th reflective element $\mathbf{Y}_{n}^{p} \in \mathbb{C}^{M \times \tau_{p}}$ received at the $\mathrm{BS}$ is

$$
\mathbf{Y}_{n}^{p}=\mathbf{h}_{n} \sum_{t=1}^{K} \sqrt{\rho_{t}} g_{t n} \boldsymbol{\psi}_{t}^{H}+\mathbf{N}_{n}^{p}
$$

where $\rho_{t}$ is the pilot power of each symbol and $\mathbf{N}_{n}^{p} \in$ $\mathbb{C}^{M \times \tau_{p}}$ is the additive noise whose elements are distributed as $C \mathcal{N}\left(0, \sigma^{2}\right)$. The channel between the $k$-th user and the BS that receives the pilot via the $n$-th reflective element is estimated from

$$
\begin{aligned}
\mathbf{y}_{k n}^{p} & =\mathbf{Y}_{n}^{p} \boldsymbol{\psi}_{k} \\
& =\sqrt{\rho_{k}} \tau_{p} \mathbf{h}_{n} g_{k n}+\tilde{\mathbf{n}}_{k n},
\end{aligned}
$$

\footnotetext{
${ }^{1}$ Although channel reciprocity is a widely adopted assumption in wireless communications, it is affected by some practical realization difficulties, which include the non-symmetric characteristics of the RF front-end circuitry at the receiver and transmitter.

${ }^{2}$ The activation of all IRS elements by using reflection patterns based on orthogonal designs, e.g., reflection patterns based on the discrete Fourier transform [10], [11], can provide higher antenna gains and reduce channel estimation errors.
}

where $\tilde{\mathbf{n}}_{k n}=\mathbf{N}_{n}^{p} \boldsymbol{\psi}_{k} \sim \mathcal{C N}\left(\mathbf{0}, \tau_{p} \sigma^{2} \mathbf{I}_{M}\right)$. By utilizing least squares estimation methods [12], the estimated channel between the $k$-th user and the BS assisted by the $n$-th reflective element can be formulated as

$$
\begin{aligned}
\tilde{\mathbf{g}}_{k n} & =\frac{1}{\sqrt{\rho_{k}} \tau_{p}} \mathbf{y}_{k n}^{p} \\
& =\mathbf{h}_{n} g_{k n}+\frac{1}{\sqrt{\rho_{k}} \tau_{p}} \tilde{\mathbf{n}}_{k n} .
\end{aligned}
$$

Given the matrix $\widetilde{\mathbf{G}}_{k}=\left[\tilde{\mathbf{g}}_{k 1}, \ldots, \tilde{\mathbf{g}}_{k N}\right] \in \mathbb{C}^{M \times N}$, the exact channel $\mathbf{G}_{k}$ between the BS and the $k$-th user is

$$
\mathbf{G}_{k}=\widetilde{\mathbf{G}}_{k}+\mathbf{E}_{k},
$$

where $\mathbf{E}_{k} \in \mathbb{C}^{M \times N}$ is the channel estimation error matrix whose individual elements are distributed as $C \mathcal{N}\left(0, \frac{\sigma^{2}}{\rho_{k} \tau_{p}}\right)$. In the sequel, we use the channel estimates in (3) to evaluate the impact of channel uncertainty on the spectral efficiency of each user.

\section{B. Downlink data transmission}

Let $\mathbf{w}_{k} \in \mathbb{C}^{M \times 1}$ and $x_{k}$, with $\mathbb{E}\left[\left|x_{k}\right|^{2}\right]=1$, be the active beamforming vector and the data symbol for the $k$-th user, respectively. In the downlink phase, each reflective element introduces a reflection coefficient to assist the communication between the BS and the users. Let $\boldsymbol{\theta}=\left[\theta_{1}, \theta_{2}, \ldots, \theta_{N}\right]^{T}$ be the coefficients, i.e., the passive beamforming vector, introduced by the IRS where $\left|\theta_{n}\right| \leq 1$ and $\arg \left(\theta_{n}\right) \in[-\pi, \pi)$ are the amplitude and the phase shift, respectively, $\forall n=1, \ldots, N$ [7]. ${ }^{3}$ The signal received by the $k$-th user can be written as

$$
y_{k}=\mathbf{g}_{k}^{H} \operatorname{diag}(\boldsymbol{\theta})^{H} \mathbf{H}^{H} \sum_{t=1}^{K} \mathbf{w}_{t} x_{t}+n_{k},
$$

where $n_{k} \sim \mathcal{C N}\left(0, \sigma^{2}\right)$ is the additive noise at the $k$-th user. Denoting $\mathbf{G}_{k}^{H}=\operatorname{diag}\left(\mathbf{g}_{k}^{*}\right) \mathbf{H}^{H} \in \mathbb{C}^{N \times M}$, we can rewrite (5) as

$$
y_{k}=\boldsymbol{\theta}^{H} \mathbf{G}_{k}^{H} \sum_{t=1}^{K} \mathbf{w}_{t} x_{t}+n_{k} .
$$

Using (4) and (6), the exact SINR at the $k$-th user, which is denoted by $\Gamma_{k}\left(\left\{\mathbf{w}_{k}\right\}, \boldsymbol{\theta}\right)$, can be written as ${ }^{4}$

$$
\Gamma_{k}\left(\left\{\mathbf{w}_{k}\right\}, \boldsymbol{\theta}\right)=\frac{\left|\boldsymbol{\theta}^{H}\left(\widetilde{\mathbf{G}}_{k}^{H}+\mathbf{E}_{k}^{H}\right) \mathbf{w}_{k}\right|^{2}}{\sum_{t=1, t \neq k}^{K}\left|\boldsymbol{\theta}^{H}\left(\widetilde{\mathbf{G}}_{k}^{H}+\mathbf{E}_{k}^{H}\right) \mathbf{w}_{t}\right|^{2}+\sigma_{k}^{2}},
$$

where $\left\{\mathbf{w}_{k}\right\}=\left\{\mathbf{w}_{1}, \mathbf{w}_{2}, \ldots, \mathbf{w}_{K}\right\}$ is the set of active beamforming vectors.

\footnotetext{
${ }^{3}$ In this letter, the amplitude and phase of the reflection coefficient of each reflecting element of the IRS can be independently optimized. Depending on the practical implementation of the reflecting elements, it may not be possible to optimize the amplitude and the phase of each reflecting element independently of each other. This is shown in e.g., [7] for a specific implementation of the reflecting elements of the IRS. The case study in [7] constitutes a valuable and promising generalization of the problem formulation analyzed in this letter, which is postponed to future research due to space limitations.

${ }^{4}$ In (7), the exact SINR is considered. This implies that the estimation error $\mathbf{E}_{k}^{H}$ appears in both the numerator and denominator of (7). In [13], on the other hand, the estimation error $\mathbf{E}_{k}^{H}$ is regarded as an additional interference term, and, therefore, it appears in the denominator of the corresponding SINR.
} 


\section{Proposed Optimization Problem}

We define the coverage probability of a user as the probability that its SINR is greater than a predefined threshold. Our aim is to jointly design the active/passive beamforming vectors so as to minimize the total transmit power while guaranteeing that the coverage probability of each user is greater than a predefined target. This problem can be formulated as follows

$$
\begin{array}{cl}
\underset{\left\{\mathbf{w}_{k}\right\}, \boldsymbol{\theta}}{\operatorname{minimize}} & \sum_{k=1}^{K} \mathbf{w}_{k}^{H} \mathbf{w}_{k} \\
\text { subject to } & \operatorname{Pr}\left(\Gamma_{k}\left(\left\{\mathbf{w}_{k}\right\}, \boldsymbol{\theta}\right) \geq \gamma_{k}\right) \geq 1-\rho_{k}, \forall k, \\
& \left|\theta_{n}\right| \leq 1, \forall n,
\end{array}
$$

where $\gamma_{k}$ and $\rho_{k}$ are the required minimum SINR to be in coverage and the predefined outage target, respectively, at the $k$-th user. Hereafter, unless otherwise stated, we assume $k \in$ $\{1, \ldots, K\}$ and $n \in\{1, \ldots, N\}$. Problem (8) is not convex due to the robust probabilistic SINR constraints. To proceed further, using (7) we recast the event $\Gamma_{k}\left(\left\{\mathbf{w}_{k}\right\}, \boldsymbol{\theta}\right) \geq \gamma_{k}$ as

$$
\frac{\left|\boldsymbol{\theta}^{H}\left(\widetilde{\mathbf{G}}_{k}^{H}+\mathbf{E}_{k}^{H}\right) \mathbf{w}_{k}\right|^{2}}{\gamma_{k}}-\sum_{\substack{t=1, t \neq k}}^{K}\left|\boldsymbol{\theta}^{H}\left(\widetilde{\mathbf{G}}_{k}^{H}+\mathbf{E}_{k}^{H}\right) \mathbf{w}_{t}\right|^{2} \geq \sigma_{k}^{2} .
$$

Defining $\mathbf{e}_{k}=\operatorname{vec}\left(\mathbf{E}_{k}\right), \mathbf{K}_{k}=\left(\boldsymbol{\theta} \boldsymbol{\theta}^{H}\right)^{T} \otimes \mathbf{B}_{k}, \mathbf{W}_{k}=\mathbf{w}_{k} \mathbf{w}_{k}^{H}$ and $\mathbf{B}_{k}=\frac{\mathbf{W}_{k}}{\gamma_{k}}-\sum_{t=1, t \neq k}^{K} \mathbf{W}_{t}$, we can rewrite (9) as

$$
\begin{aligned}
& \boldsymbol{\theta}^{H}\left(\widetilde{\mathbf{G}}_{k}^{H}+\mathbf{E}_{k}^{H}\right) \mathbf{B}_{k}\left(\widetilde{\mathbf{G}}_{k}+\mathbf{E}_{k}\right) \boldsymbol{\theta} \geq \sigma_{k}^{2}, \\
\Leftrightarrow & \operatorname{tr}\left(\left(\widetilde{\mathbf{G}}_{k}^{H}+\mathbf{E}_{k}^{H}\right) \mathbf{B}_{k}\left(\widetilde{\mathbf{G}}_{k}+\mathbf{E}_{k}\right) \boldsymbol{\theta} \boldsymbol{\theta}^{H}\right) \geq \sigma_{k}^{2}, \\
\Leftrightarrow & \operatorname{vec}\left(\widetilde{\mathbf{G}}_{k}+\mathbf{E}_{k}\right)^{H} \operatorname{vec}\left(\mathbf{B}_{k}\left(\widetilde{\mathbf{G}}_{k}+\mathbf{E}_{k}\right) \boldsymbol{\theta} \boldsymbol{\theta}^{H}\right) \geq \sigma_{k}^{2}, \\
\Leftrightarrow & \operatorname{vec}\left(\widetilde{\mathbf{G}}_{k}+\mathbf{E}_{k}\right)^{H} \mathbf{K}_{k} \operatorname{vec}\left(\widetilde{\mathbf{G}}_{k}+\mathbf{E}_{k}\right) \geq \sigma_{k}^{2}, \\
\Leftrightarrow & \mathbf{e}_{k}^{H} \mathbf{K}_{k} \mathbf{e}_{k}+2 \operatorname{Re}\left\{\mathbf{e}_{k}^{H} \mathbf{K}_{k} \operatorname{vec}\left(\widetilde{\mathbf{G}}_{k}\right)\right\} \geq d_{k} .
\end{aligned}
$$

To obtain (11) from (10), the identity $\mathbf{a}^{H} \mathbf{B a}=\operatorname{Tr}\left(\mathbf{B a a}^{H}\right)$ is used, and to obtain (13) from (12), the identity vec $(\mathbf{A Y B})=$ $\left(\mathbf{B}^{T} \otimes \mathbf{A}\right) \operatorname{vec}(\mathbf{Y})$ is used. By defining the following function that accounts for the channel estimation error of the $k$-th user

$$
f\left(\mathbf{e}_{k}\right)=\mathbf{e}_{k}^{H} \mathbf{K}_{k} \mathbf{e}_{k}+2 \operatorname{Re}\left\{\mathbf{e}_{k}^{H} \mathbf{K}_{k} \operatorname{vec}\left(\widetilde{\mathbf{G}}_{k}\right)\right\},
$$

problem (8) can be equivalently formulated as

$$
\begin{array}{ll}
\underset{\left\{\mathbf{W}_{k}\right\}, \boldsymbol{\theta}}{\operatorname{minimize}} & \operatorname{tr}\left(\sum_{k=1}^{K} \mathbf{W}_{k}\right) \\
\text { subject to } & \operatorname{Pr}\left(f\left(\mathbf{e}_{k}\right) \geq d_{k}\right) \geq 1-\rho_{k}, \forall k, \\
& \operatorname{rank}\left(\mathbf{W}_{k}\right)=1, \forall k, \\
& \left|\theta_{n}\right| \leq 1, \forall n .
\end{array}
$$

In (16), we have introduced the set of matrices $\left\{\mathbf{W}_{k}\right\}=$ $\left\{\mathbf{W}_{1}, \mathbf{W}_{2}, \ldots, \mathbf{W}_{K}\right\}$ and $d_{k}=\sigma_{k}^{2}-\operatorname{vec}\left(\widetilde{\mathbf{G}}_{k}\right)^{H} \mathbf{K}_{k} \operatorname{vec}\left(\widetilde{\mathbf{G}}_{k}\right)$. We note that problem (16) is still non-convex due to the inherent non-convexity of the probabilistic SINR and the rank constraints. We first handle the probabilistic SINR constraints by introducing the following lemma.
Lemma 1. For any $\eta_{k}>0$, the statement below holds true:

$$
\operatorname{Pr}\left(f\left(\mathbf{e}_{k}\right) \geq \Upsilon\left(\eta_{k}\right)\right) \geq 1-e^{-\eta_{k}},
$$

where $\Upsilon\left(\eta_{k}\right)$ is defined as

$$
\begin{aligned}
\Upsilon\left(\eta_{k}\right) & =-\sqrt{2 \eta_{k}} \sqrt{\frac{\sigma^{4}}{\rho_{k}^{2} \tau_{p}^{2}}\left\|\mathbf{K}_{\mathbf{k}}\right\|_{F}^{2}+\frac{2 \sigma^{2}}{\rho_{k} \tau_{p}}\left\|\mathbf{K}_{k} \operatorname{vec}\left(\widetilde{\mathbf{G}}_{k}\right)\right\|^{2}} \\
& +\frac{\sigma^{2}}{\rho_{k} \tau_{p}} \operatorname{tr}\left(\mathbf{K}_{k}\right)-\frac{\eta_{k} \sigma^{4}}{\rho_{k}^{2} \tau_{p}^{2}} \lambda^{+}\left(\mathbf{K}_{k}\right)
\end{aligned}
$$

and $\lambda^{+}\left(\mathbf{K}_{k}\right)=\min \left\{\lambda_{\min }\left(\mathbf{K}_{k}\right), 0\right\}$ where $\lambda_{\min }\left(\mathbf{K}_{k}\right)$ is the minimum eigenvalue of $\mathbf{K}_{k}$.

Proof. The proof is obtained by applying the Bernsteintype inequality [9] to the Gaussian random variable $\mathbf{e}_{k} \sim$ $C \mathcal{N}\left(\mathbf{0}, \frac{\sigma^{2}}{\rho_{k} \tau_{p}} \mathbf{I}_{M N}\right)$ with the positive definite matrix $\frac{\sigma^{2}}{\rho_{k} \tau_{p}} \mathbf{K}_{k}$ and the vector $\frac{\sigma}{\sqrt{\rho_{k} \tau_{p}}} \mathbf{K}_{k} \operatorname{vec}\left(\widetilde{\mathbf{G}}_{k}\right)$ in a standard quadratic form.

By setting $\eta_{k}=-\ln \rho_{k}$ and using Lemma 1, the probabilistic constraint in (16) can be rewritten as

$$
\begin{aligned}
& -\sqrt{2 \ln \left(1 / \rho_{k}\right)} \sqrt{\frac{\sigma^{4}}{\rho_{k}^{2} \tau_{p}^{2}}\left\|\mathbf{K}_{k}\right\|_{F}^{2}+\frac{2 \sigma^{2}}{\rho_{k} \tau_{p}}\left\|\mathbf{K}_{k} \operatorname{vec}\left(\widetilde{\mathbf{G}}_{k}\right)\right\|^{2}} \\
& +\frac{\sigma^{2}}{\rho_{k} \tau_{p}} \operatorname{tr}\left(\mathbf{K}_{k}\right)-\frac{\ln \left(1 / \rho_{k}\right) \sigma^{4}}{\rho_{k}^{2} \tau_{p}^{2}} \lambda^{+}\left(\mathbf{K}_{k}\right) \geq d_{k} .
\end{aligned}
$$

We introduce two auxiliary variables $\mu_{k}$ and $v_{k}$ to equivalently cast (19) as the following series of inequalities

$$
\begin{array}{r}
\frac{\sigma^{2}}{\rho_{k} \tau_{p}} \operatorname{tr}\left(\mathbf{K}_{k}\right)-\sqrt{2 \ln \left(1 / \rho_{k}\right)} \mu_{k}-\frac{\ln \left(1 / \rho_{k}\right) \sigma^{4}}{\rho_{k}^{2} \tau_{p}^{2}} v_{k} \\
\geq d_{k}, \\
\sqrt{\frac{\sigma^{4}}{\rho_{k}^{2} \tau_{p}^{2}}\left\|\mathbf{K}_{\mathbf{k}}\right\|_{F}^{2}+\frac{2 \sigma^{2}}{\rho_{k} \tau_{p}}\left\|\mathbf{K}_{k} \operatorname{vec}\left(\widetilde{\mathbf{G}}_{k}\right)\right\|^{2}} \leq \mu_{k}, \\
v_{k} \mathbf{I}_{M N}+\mathbf{K}_{k} \geq \mathbf{0}, \\
v_{k} \geq 0 .
\end{array}
$$

While the constraints (20), (22), and (23) adhere to the standard form of a semi-definite program with respect to $\mathbf{K}_{k}$, we can reformulate (21) in a standard second-order-cone (SOC) constraint as

$$
\left\|\left[\begin{array}{c}
\frac{\sqrt{2} \sigma}{\sqrt{\rho_{k} \tau_{p}}} \mathbf{K}_{k} \operatorname{vec}\left(\widetilde{\mathbf{G}}_{k}\right) \\
\frac{\sigma^{2}}{\rho_{k} \tau_{p}} \operatorname{vec}\left(\mathbf{K}_{k}\right)
\end{array}\right]\right\| \leq \mu_{k} .
$$

To proceed further, we introduce a new variable $\boldsymbol{\Theta}=\boldsymbol{\theta} \boldsymbol{\theta}^{H}$ and recast (16) as

$$
\begin{array}{ll}
\underset{\left\{\mathbf{W}_{k}\right\}, \boldsymbol{\Theta},\left\{\boldsymbol{\mu}_{k}\right\},\left\{v_{k}\right\}}{\operatorname{minimize}} & \operatorname{tr}\left(\sum_{k=1}^{K} \mathbf{W}_{k}\right) \\
\text { subject to } & (20),(22),(23),(24), \forall k, \\
& \mathbf{W}_{k} \geq \mathbf{0}, \forall k, \\
& \operatorname{rank}\left(\mathbf{W}_{k}\right)=1, \forall k, \\
& \operatorname{diag}(\boldsymbol{\Theta}) \leq \mathbf{1}_{N}, \\
& \boldsymbol{\Theta} \geq \mathbf{0}, \\
& \operatorname{rank}(\boldsymbol{\Theta})=1 .
\end{array}
$$


By analyzing the constraints (20), (22) and (24), we observe that they are non-convex with respect to $\left\{\mathbf{W}_{k}\right\}$ and $\boldsymbol{\Theta}$. These constraints, in fact, depend on $\mathbf{K}_{k}$, which is a function of $\left\{\mathbf{W}_{k}\right\}$ and $\boldsymbol{\Theta}$. To overcome this issue, we use alternating optimization so as to obtain a fixed-point solution of problem (25). In particular, starting from an initial value of the reflection coefficients $\boldsymbol{\Theta}^{(0)}$ by replacing $\mathbf{W}_{k}, \mu_{k}$, and $v_{k}$, respectively, with $\mathbf{W}_{k}^{(i)}$, $\mu_{k}^{(i)}$, and $v_{k}^{(i)}, \forall k$, we solve the following subproblem during the $i$-th iteration

$$
\begin{array}{ll}
\underset{\left\{\mathbf{W}_{k}^{(i)}\right\},\left\{\mu_{k}^{(i)}\right\},\left\{v_{k}^{(i)}\right\}}{\operatorname{minimize}} & \operatorname{tr}\left(\sum_{k=1}^{K} \mathbf{W}_{k}^{(i)}\right) \\
\text { subject to } & (20),(22),(23),(24), \forall k, \\
& \mathbf{W}_{k}^{(i)} \geq \mathbf{0}, \forall k, \\
& \operatorname{rank}\left(\mathbf{W}_{k}^{(i)}\right)=1, \forall k .
\end{array}
$$

Problem (26) is still non-convex due to the rank-one constraint on $\mathbf{W}_{k}^{(i)}$. However, problem (26) has a structure similar to that in [14]. Hence, we can exploit the same methodology as in [14, Theorem 2] to show that its optimal solution is rankone if problem (26) is feasible. Consequently, problem (26) is equivalent to the following LMI form

$$
\begin{array}{ll}
\underset{\left\{\mathbf{W}_{k}^{(i)}\right\},\left\{\mu_{k}^{(i)}\right\},\left\{v_{k}^{(i)}\right\}}{\operatorname{minimize}} & \operatorname{tr}\left(\sum_{k=1}^{K} \mathbf{W}_{k}^{(i)}\right) \\
\text { subject to } & (20),(22),(23),(24), \forall k, \\
& \mathbf{W}_{k}^{(i)} \geq \mathbf{0}, \forall k,
\end{array}
$$

which yields the optimal solution $\left\{\mathbf{W}_{k}^{(i)}\right\},\left\{\mu_{k}^{(i)}\right\}$ and $\left\{v_{k}^{(i)}\right\}$ for a given matrix $\boldsymbol{\Theta}^{(i-1)}$. Since (27) is a semidefinite program, its optimal solution is obtained in polynomial time by using general purpose interior-point toolboxes, e.g., CVX [15]. After obtaining $\left\{\mathbf{W}_{k}^{(i)}\right\},\left\{\mu_{k}^{(i)}\right\}$ and $\left\{v_{k}^{(i)}\right\}$ from the solution of (27), the matrix $\Theta^{(i)}$ is attained by solving the following feasibility problem

$$
\begin{array}{ll}
\text { find } & \boldsymbol{\Theta}^{(i)} \\
\text { subject to } & (20),(22),(24), \forall k, \\
& \operatorname{diag}\left(\boldsymbol{\Theta}^{(i)}\right) \leq \mathbf{1}_{N}, \\
& \boldsymbol{\Theta}^{(i)} \geq \mathbf{0} \\
& \operatorname{rank}\left(\boldsymbol{\Theta}^{(i)}\right)=1 .
\end{array}
$$

Problem (28) is non-convex due to the rank-one constraint on $\boldsymbol{\Theta}^{(i)}$. To tackle this problem, we propose to solve the following problem

$$
\begin{array}{cl}
\underset{\boldsymbol{\Theta}^{(i)}}{\operatorname{minimize}} & \operatorname{tr}\left(\boldsymbol{\Theta}^{(i)}\right) \\
\text { subject to } & (20),(22),(24), \forall k, \\
& \operatorname{diag}\left(\boldsymbol{\Theta}^{(i)}\right) \leq \mathbf{1}_{N}, \\
& \boldsymbol{\Theta}^{(i)} \geq \mathbf{0},
\end{array}
$$

whose global optimum can be obtained by using CVX. Exploiting the same methodology as in [14, Theorem 2], we can show that problem (29) yields a rank-one optimal solution. Therefore, we can conclude that problem (29) is an approximation of problem (28), i.e., every feasible solution of
Algorithm 1 Alternating optimization to solve (25)

Input: Channel estimate matrices $\widetilde{\mathbf{G}}_{k}, \forall k$; Initial phase shift coefficients $\Theta^{(0)}$; Tolerance value $\delta$; Set $i=0$ and initial the cost function $C^{(0)}=0$.

1. $i=i+1$; Update $\left\{\mathbf{W}_{k}^{(i)}\right\},\left\{\mu_{k}^{(i)}\right\}$, and $\left\{v_{k}^{(i)}\right\}$ for all $k$, by solving problem (27) where every $\theta_{n}^{(i-1)}$ is computed from the previous iteration.

2. Update $\boldsymbol{\Theta}_{k}^{(i)}$ by solving problem (29) where $\left\{\mathbf{W}_{k}^{(i)}\right\}$, $\left\{\mu_{k}^{(i)}\right\}$, and $\left\{v_{k}^{(i)}\right\}$ are obtained from Step 1.

3. Compute the cost function $C^{(i)}=\operatorname{tr}\left(\sum_{k=1}^{K} \mathbf{W}_{k}^{(i)}\right)$.

4. Check the stopping criterion: If $\left|C^{(i)}-C^{(i-1)}\right| \leq \delta \rightarrow$ Stop. Otherwise, repeat Steps $1-3$.

Output: The fixed point solution: $\left\{\mathbf{W}_{k}^{(i)}\right\}$ and $\boldsymbol{\Theta}^{(i)}$.

(29) is also feasible for (28), see e.g. [14, Section III.B] and references therein.

By direct inspection, subproblems (27) and (29) are convex and their feasible domains are convex sets. Therefore, the proposed iterative algorithm converges to a fixed-point solution [16] when the subproblems (27) and (29) are feasible. The proposed alternating optimization method is summarized in Algorithm 1.5

Finally, the active/passive beamforming vectors $\mathbf{w}_{k}$ and $\boldsymbol{\theta}$ are, respectively, as follows $\sqrt{\lambda_{k}^{(w)}} \mathbf{z}_{k}^{(w)}$ and $\sqrt{\lambda^{(\theta)}} \mathbf{z}^{(\theta)}$, where $\lambda_{k}^{(w)}$ and $\lambda^{(\theta)}$ are the non-zero eigenvalues, and $\mathbf{z}_{k}^{(w)}$ and $\mathbf{z}^{(\theta)}$ are the corresponding eigenvectors of the rank-one fixed-point solution $\mathbf{W}_{k}^{(i)}$ and $\boldsymbol{\Theta}^{(i)}$, respectively [14, Section IV.A].

\section{NumericAl RESUlts}

We consider a MISO system in the absence of light-ofsight so that there is no direct path from the BS to the users. The 3GPP Urban Micro channel model is used [17]. In the coverage area, the users are randomly distributed, but the minimum distance to the IRS is $10 \mathrm{~m}$. The distance between the BS and the IRS is $80 \mathrm{~m}$. The large-scale fading coefficient of the point-to-point link between the BS and the IRS is 1. There are 15 elements at the IRS. The noise variance is $-96 \mathrm{dBm}$. The bandwidth is $10 \mathrm{MHz}$. The large-scale fading coefficient between the user $k$ and the IRS is defined as

$$
\beta_{k}[\mathrm{~dB}]=-15.1-26 \log _{10}\left(f_{c}\right)-37.6 \log _{10}\left(d_{k} / 1 \mathrm{~m}\right),
$$

where $f_{c}=3 \mathrm{GHz}$ is the carrier frequency, $d_{k}$ is the distance between the user $k$ and the IRS $\left(d_{k} \geq 10 \mathrm{~m}\right)$. The SINR requirement of each user is $4 \mathrm{~dB}$ and the outage probability is 0.1 .

Fig. 1 illustrates the convergence of the proposed algorithm as a function of the number of users. We observe that Algorithm 1 converges to a fixed-point solution after less than 5 iterations in all tested cases. These numerical results confirm the statement about the convergence of the proposed algorithm.

\footnotetext{
${ }^{5}$ Although global optimality can be obtained by solving subproblems (27) and (29) in each iteration, the global optimal solution of the original problem (25) may not be attained due to the inherent non-convexity of (25). In fact, the proposed algorithm yields a suboptimal solution to the original non-convex problem.
} 


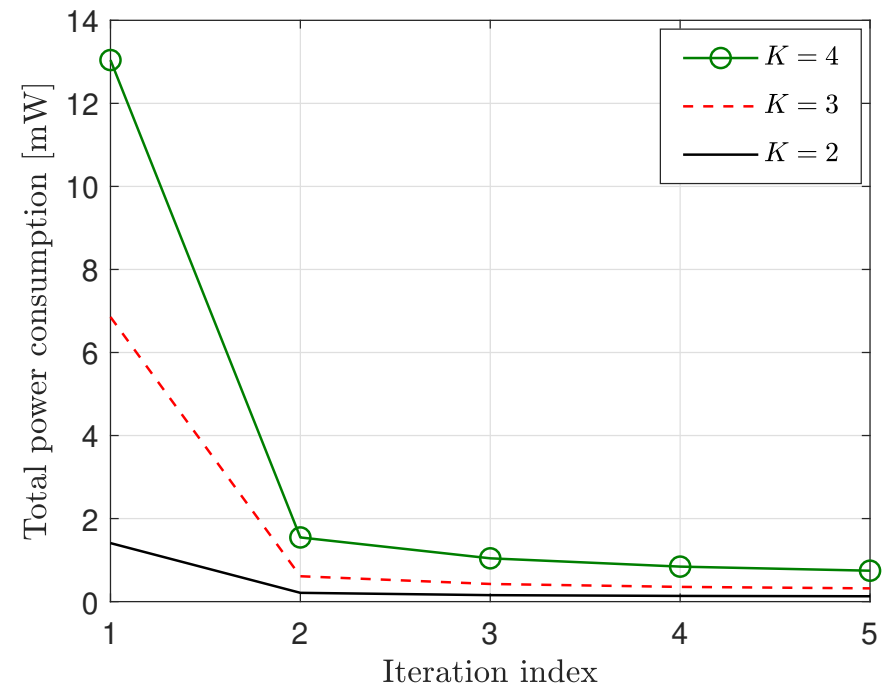

Fig. 1: Convergence of Algorithm 1 for a different number of users.

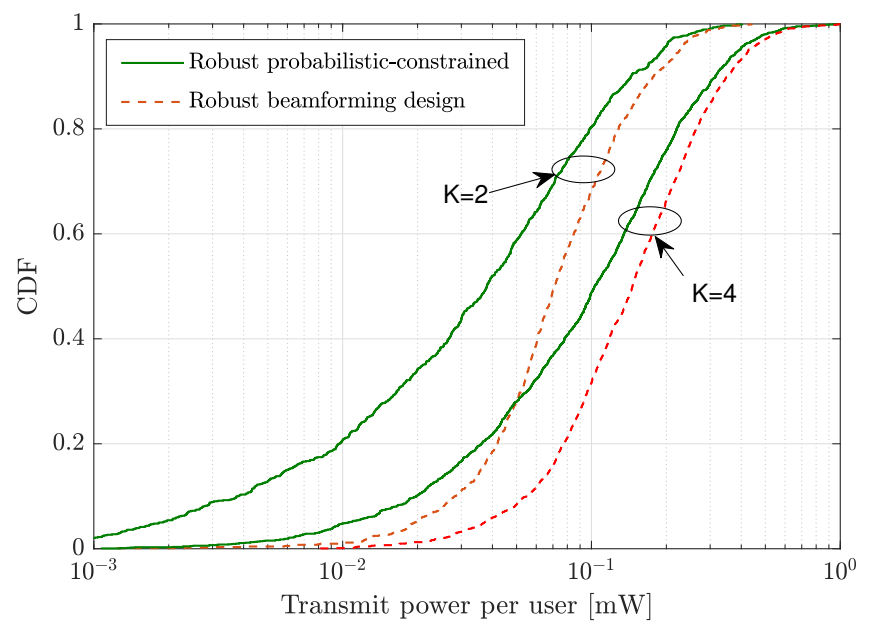

Fig. 2: Transmit power consumption per user [mW].

Fig. 2 illustrates the transmit power per user of the proposed approach, i.e., Algorithm 1, which is denoted as "Robust probabilistic-constrained", and the benchmark method in [8], which is denoted as "Robust beamforming design". It can be observed that the proposed approach consumes $66 \%$ and $28 \%$ less power than the benchmark method in [8] when the system serves 2 users and 4 users, respectively. This is due to the fact that the benchmark method in [8] allocates extra resources to protect rarely occurring worst-case events while the proposed approach allows the QoS constraints to be violated with some non-zero probabilities.

Due to the increase of the mutual interference, Fig. 2 shows that the system must allocate more power to each user when the number of coexisting users increases. For example, the proposed approach requires approximately $0.06 \mathrm{~mW}$ in order to guarantee the SINR requirements with an outage probability of 0.1 when 2 users are in the coverage area. On the other hand, the power allocated to each user increases up to $0.14 \mathrm{~mW}$ if 4 users need to be served.

\section{CONCLUSION}

We have formulated and solved a robust probabilisticconstrained optimization problem for IRS-aided MISO communication systems in order to tackle imperfect estimates of the CSI. The optimal beamforming vectors at the BS and the reflecting elements at the IRS are iteratively computed via a converging alternating optimization algorithm. Numerical results reveal a fast convergent behavior of the proposed algorithm, i.e., within a few iterations. The results confirm the superior performance of the proposed approach compared with a benchmark method.

\section{REFERENCES}

[1] Q. Wu and R. Zhang, "Towards smart and reconfigurable environment: Intelligent reflecting surface aided wireless network," IEEE Communications Magazine, vol. 58, no. 1, pp. 106-112, January 2020.

[2] M. Di Renzo, A. Zappone, M. Debbah, M.-S. Alouini, C. Yuen, J. de Rosny, and S. Tretyakov, "Smart radio environments empowered by reconfigurable intelligent surfaces: How it works, state of research, and road ahead," arXiv preprint arXiv:2004.09352, 2020.

[3] M. Di Renzo, M. Debbah, D.-T. Phan-Huy, A. Zappone, M.-S Alouini, C. Yuen, V. Sciancalepore, G. C Alexandropoulos, J. Hoydis, H. Gacanin, J. De Rosny, A. Bounceur, G. Lerosey, and M. Fink, "Smart radio environments empowered by reconfigurable AI metasurfaces: An idea whose time has come," EURASIP Journal on Wireless Communications and Networking, vol. 2019, no. 1, pp. 1-20, 2019.

[4] E. Basar, M. Di Renzo, J. De Rosny, M. Debbah, M. Alouini, and R. Zhang, "Wireless communications through reconfigurable intelligent surfaces," IEEE Access, vol. 7, pp. 116 753-116773, 2019.

[5] X. Qian, M. Di Renzo, J. Liu, A. Kammoun, and M.-S. Alouini, "Beamforming through reconfigurable intelligent surfaces in singleuser MIMO systems: SNR distribution and scaling laws in the presence of channel fading and phase noise," 2020, [Online]. Available: https://arxiv.org/pdf/2005.07472.pdf.

[6] A. Zappone, M. Di Renzo, F. Shams, X. Qian, and M. Debbah, "Overhead-aware design of reconfigurable intelligent surfaces in smart radio environments," 2020, [Online]. Available: https://arxiv.org/abs/2003.02538.

[7] S. Abeywickrama, R. Zhang, Q. Wu, and C. Yuen, "Intelligent reflecting surface: Practical phase shift model and beamforming optimization," 2020, [Online]. Available: https://arxiv.org/pdf/2002.10112.pdf.

[8] G. Zhou, C. Pan, H. Ren, K. Wang, M. Di Renzo, and A. Nallanathan, "Robust beamforming design for intelligent reflecting surface aided MISO communication system," 2019. [Online]. Available: https://arxiv.org/pdf/1911.06237.pdf

[9] I. Bechar, "A Bernstein-type inequality for stochastic processes of quadratic forms of gaussian variables," 2009, [Online]. Available: http://arxiv.org/pdf/0909.3595.pdf.

[10] B. Zheng and R. Zhang, "Intelligent reflecting surface-enhanced OFDM: Channel estimation and reflection optimization," IEEE Wireless Commun. Lett., vol. 9, no. 4, pp. 518-522, 2019.

[11] B. Zheng, C. You, and R. Zhang, "Intelligent reflecting surface assisted multi-user OFDMA: Channel estimation and training design," arXiv preprint arXiv:2003.00648, 2020.

[12] S. Kay, Fundamentals of Statistical Signal Processing: Estimation Theory. Prentice Hall, 1993.

[13] C. You, B. Zheng, and R. Zhang, "Channel estimation and passive beamforming for intelligent reflecting surface: Discrete phase shift and progressive refinement," IEEE J. Sel. Areas Commun., Available: http://arxiv. org/abs.

[14] T. A. Le, Q.-T. Vien, H. X. Nguyen, D. W. K. Ng, and R. Schober, "Robust chance-constrained optimization for power-efficient and secure SWIPT systems," IEEE Trans. Green Commun. Netw., vol. 1, no. 3, pp. 333-346, 2017.

[15] CVX Research Inc., "CVX: Matlab software for disciplined convex programming, academic users," http://cvxr.com/cvx, 2015.

[16] T. Van Chien, E. Björnson, and E. G. Larsson, "Joint pilot design and uplink power allocation in multi-cell Massive MIMO systems," IEEE Trans. Wireless Commun., vol. 17, no. 3, pp. 2000-2015, 2018.

[17] Further advancements for E-UTRA physical layer aspects (Release 9). 3GPP TS 36.814, Mar. 2010. 